\title{
Elaboración de un curso de formación sobre la gestión clínica y metodológica del paciente renal
}

\author{
Marta Pérez Doctor, Trinidad López Sánchez, Jesús Zambrana Hervás, Olga Vanesa Caballero Rubio, Mª \\ Luz Amoedo Cabrera, M ${ }^{\mathrm{a}}$ Carmen Chozas López
}

Hospital Universitario Virgen del Rocío. Sevilla

\section{Introducción:}

Se ha elaborado un curso de formación dirigido a los profesionales de enfermería con la intención de mejorar sus conocimientos sobre la patología renal e incrementar la calidad asistencial, de modo que desarrollen unas aptitudes básicas para influir positivamente en el tratamiento, en los cuidados, en la resolución de los diagnósticos de enfermería más prevalentes en los enfermos renales y promocionen hábitos de vida saludable a través de la educación sanitaria.

\section{Objetivo general:}

- Elaboración un curso de formación sobre la gestión clínica y metodológica del paciente renal.

\section{Objetivos específicos:}

- Actualización de conocimientos en anatomía, fisiología y patología renal, así como patologías asociadas a los tratamientos sustitutivos.

- Adquisición de contenidos procedimentales focalizados en los tratamientos sustitutivos: hemodiálisis, diálisis peritoneal y trasplante.

- Actualización de los cuidados de enfermería al paciente renal basado en valoración integral focaliza$\mathrm{da}$, planes de cuidados estandarizados y recomendaciones.

\section{Metodología:}

- Basada en la investigación, a partir de la concepción constructivista del aprendizaje.

- La programación de la unidad didáctica consta de unos elementos básicos que servirán de guía para nuestras actividades docentes y que hemos aplicado al aprendizaje de la gestión clínica y metodológica de los cuidados al paciente renal.

- Evidencia interna de las docentes, con más de diez años de experiencia profesional y el CAP.

\section{Resultados:}

1. Tema principal y contextualización:

- Actualización de cuidados de enfermería en el paciente renal y desarrollo de actitudes y aptitudes básicas en los profesionales objeto del aprendizaje con el fin de mejorar los resultados asistenciales al paciente renal.

- Dirigido a todos los profesionales de enfermería, especialmente a los que se dedican al área médicoquirúrgica, tanto en centros públicos, privados como concertados.

2. Objetivos didácticos referenciales relacionados con los contenidos: Se ha elaborado un temario dividido en ocho unidades didácticas íntimamente relacionadas con los objetivos:

- Anatomía y fisiología renal.

- Patología renal. 
- Tratamientos sustitutivos en la insuficiencia renal.

- Hemodiálisis.

- Diálisis peritoneal.

- Nutrición en la insuficiencia renal.

- Patologías asociadas en el paciente renal con insuficiencia renal crónica terminal (IRCT) en tratamiento sustitutivo.

- Trasplante renal.

3. Metodología y actividades de enseñanza/aprendizaje:

- Exposición del contenido teórico con apoyo audiovisual y muestra de material especifico de diálisis.

4. Temporalización:

- Curso presencial, de 20 horas de duración, repartidas en cuatro horas durante cinco días.

- El $40 \%$ del tiempo dedicado al aprendizaje de conceptos, actitudes y aptitudes, y el $60 \%$ a los procedimientos.

5. Recursos necesarios y disponibles:

- Aula bien equipada.

- Ordenador con conexión a internet.

- Material teórico y práctico específico de diálisis y trasplante.

6. Criterios de evaluación:

- Evaluación inicial y final con cuestionario de 12 preguntas de respuestas múltiples de todo el temario, donde sólo una de ellas es correcta.

\section{Conclusiones:}

- La formación basada en la investigación y estructurada como una unidad didáctica aporta un esquema adecuado a la necesidad formativa del alumno.

- Los cursos adecuadamente estructurados incrementan los conocimientos, actitudes y aptitudes básicos del profesional de enfermería incrementando la calidad asistencial al paciente renal.

\section{Referencias Bibliográficas}

1. II Plan de Calidad 2005-2008. Consejería de salud, Junta de Andalucía.
2. Estrategias de mejora de los cuidados enfermeros en el Servicio Andaluz de Salud. Coordinación Regional de Desarrollo e Innovación de Cuidados. Abril 2004.

3. J.Martín Toscano, J.Correa Manfredi, T.Paniagua Aguilar y cols. Didáctica General. Materiales Didácticos.CAP 2006.Instituto de Ciencias de la Educación ICE. Universidad de Sevilla ATRIL.

4. J.A.Atienza Ruiz. Didáctica Especial de las Ciencias de la Salud. ICE Universidad de Sevilla. CAP 2006.

5. La Metodología Enfermera. Observatorio. FUDEN (Fundación para el desarrollo de la Enfermería). http://www.ome.es/index.cfm.

6. Servicio Andaluz de Salud. Gestión por Procesos Asistenciales Integrados. http://www.juntadeandalucia.es/salud/principal/documentos.asp? $\mathrm{id}=608$ [Visitado el 05/03/2007].

7. Proceso Asistencial. Tratamiento Sustitutivo de la Insuficiencia Renal Crónica: Diálisis y Trasplante. SAS. Conserjería de Salud. Junta de Andalucía. Sevilla. 2005.

8. F. Coronel, J. Montenegro, R. Selgas, 0. Celadilla, M. Tejuca. 2005.Manual de Diálisis Peritoneal. SEDEN 2005.

9. NANDA: Diagnósticos Enfermeros: definición y clasificaciones 2005-2006. Revisión por Luis Rodrigo, MT. Edición 2005. Editorial Elsevier.

10. Clasificación de Intervenciones de Enfermería. (NIC).Cuarta Edición (Cobos Serrano, JL.y cols.. 2005).Mccloskey, J. Bulechek. Editorial Elsevier.

11. Clasificación de Resultados de Enfermería (NOC). Tercera Edición 2005 (Cobos Serrano, JL. Y cols. Ed. Elsevier.

12. I.Paneque Sánchez-T.Memorial funcional. Gestión por Procesos Asistenciales. H.U.Virgen del Rocío. Febrero 2003.

13. Andres, J; Fortuny, C. Cuidados de enfermería en la insuficiencia renal. Ed ELA, 1994, Madrid.

14. Andreu Periz, L; Force Sanmartin, E. 500 cuestiones que plantea el cuidado del enfermo renal. Ed Elsevier Masson, $2^{a}$ edición, 2001. 